\title{
One-particle reducibility in effective scattering theory
}

\author{
V. Vereshagin * \\ Saint-Petersburg State University, 198504 St.-Petersburg, Russia
}

\begin{abstract}
To construct the reasonable renormalization scheme suitable for the effective theories one needs to resolve the "problem of couplings" because the number of free parameters in a theory should be finite. Otherwise the theory would loose its predictive power. In the case of effective theory already the first step on this way shows the necessity to solve the above-mentioned problem for the 1-loop 2-leg function traditionally called self energy. In contrast to the customary renormalizable models the corresponding Feynman graph demonstrates divergencies that require introducing of an infinite number of prescriptions. In the recent paper [1] it has been shown that the way out of this difficulty requires the revision of the notion of one-particle reducibility. The point is that in effective scattering theory one can introduce two different notions: the graphic reducibility and the analytic one. Below we explain the main ideas of the paper [1] and recall some notions and definitions introduced earlier in [2] and [3].
\end{abstract}

\section{Introduction}

First of all, it should be recalled that the field theory is said to be effective if:

1. It is based on the Dyson formula for S-matrix elements ( $\mathrm{f}$ and $\mathrm{i}$ stand for the final and initial states, respectively):

$$
S_{f i}=\left\langle f\left|T_{W} \exp \left\{i \int L_{\text {int }} d x\right\}\right| i\right\rangle .
$$

The symbol $T_{W}$ in this formula denotes Wick's (manifestly covariant) T-product. The noncovariant terms in the interaction Lagrangian and in propagators are to be discarded (see [4], [5]). In the case of effective theories only discussed below this does not lead to any uncertainties.

2. The interaction Lagrangian is defined as the interaction picture operator; it contains all the monomials consistent with the given algebraic symmetry.

It is clear that the effective field theory is renormalizable: all necessary counterterms are presented in the Lagrangian by construction. This fact, however, does not make life easier because one needs to formulate an infinite number of renormalization prescriptions (RP's) in order to fix the finite parts of relevant counterterms. This (so-called "problem of couplings") is the main obstacle on the way of constructing the physically meaningful theory of S matrix.

^E-mail: vvv@av2467.spb.edu 
As known (see, e.g. [4]), the full set of parameters that appear in the effective field theory can be divided into two independent subsets: the essential parameters (those independent combinations of the Lagrangian coupling constants which the S-matrix elements depend upon) and the redundant ones (those needed to calculate the Green functions off the mass shell). As long as we are only interested in finiteness and physical definiteness of S-matrix elements (amplitudes of the physical processes) there is no necessity in formulating the RP's for redundant parameters. It is this scheme (called in [3] the effective scattering theory) which we discuss below.

To make use of this idea one needs to point out the full list of essential parameters. Here two notes are in order. First, as shown in [2] (see also [3]), the sum of S-matrix graphs of the given loop order L depends on the Lth level resultant parameters which are the only numbers to be fixed in the process of step-by-step renormalization. Second, the list of those resultant parameters may be further shortened if certain additional restrictions (say, polynomial boundedness requirements) are imposed. Strictly speaking, only the parameters from that shortened list should be called essential. For this reason the combinations that appear before attracting the additional conditions like the polynomial boundedness are called resultant.

There is an obvious problem that precludes constructing the renormalization scheme completely formulated in terms of S matrix (on shell) parameters: we need to know the two leg function (so-called self energy) at arbitrary value of $p^{2}$, not only on the mass shell. In the paper [1] it has been shown that this problem turns out quite solvable if one redefines the notion of one-particle irreducibility (1PI). Below we explain this point in terms of the single scalar effective scattering theory.

\section{Preliminary notes and definitions}

First we would like to recall some definitions and notions discussed earlier in [2], [3] and [1].

We work in terms of $(4 n-10)$ scalar variables needed to describe the $n$-leg full effective vertex $V\left(p_{1}, \ldots, p_{n}\right)$ with external lines marked by their (off shell!) momenta $p_{1}, \ldots, p_{n}$ and depending on $(4 n-10)$ scalar variables

$$
\left[\kappa_{1}, \ldots, \kappa_{n} ; v_{1}, \ldots, v_{3 n-10}\right]
$$

Here

$$
\kappa_{k}=p_{k}^{2}-m^{2} \equiv \kappa\left(p_{k}\right)
$$

(below we call the variables of this kind as the shell deviations; note that these are the only variables which the triple effective vertex may depend upon) and

$$
v_{r} \equiv \sum_{i, j=1}^{n} s_{i j}^{r}\left(p_{i} \cdot p_{j}\right) \quad(r=1, \ldots, 3 n-10)
$$

stand for the independent scalar products $\left(s_{i j}^{r}\right.$ are the relevant numerical coefficients). Since the effective vertex is just a series in scalar variables $\kappa_{i}(i=1, \ldots, n)$ and $v_{k}(k=1, \ldots, 3 n-10)$, it can be written as follows

$$
V\left(p_{1}, \ldots, p_{n}\right) \equiv V\left(\kappa_{1}, \ldots, \kappa_{n} ; v_{1}, \ldots, v_{3 n-10}\right)
$$

$$
=\sum_{i_{1}, i_{n}=0}^{\infty} V_{i_{1}, \ldots, i_{n}}\left(v_{1}, \ldots, v_{3 n-10}\right) \kappa_{1}{ }^{i_{1}} \ldots \kappa_{n}{ }^{i_{n}} .
$$

The item

$$
V_{0, \ldots, 0}\left(v_{1}, \ldots, v_{3 n-10}\right)
$$


is called minimal vertex or, the same, vertex minimal with respect to all its lines. It contrast to the full effective vertex (5) it does not contain factors $p_{i}^{2}-m^{2}$.

To better understand the importance of this notion it is instructive to consider the 4-leg tree graph $\Gamma$ constructed from two full 3-leg effective vertices, say, $V\left(p_{1}, p_{2}, k\right)$ and $V\left(p_{3}, p_{4}, k\right)$ connected to one another by the propagator line $k$ :

$$
\Gamma\left(p_{1}, \ldots, p_{4}\right)=V\left(p_{1}, p_{2}, k\right) \frac{1}{k^{2}-m^{2}} V\left(p_{3}, p_{4}, k\right) .
$$

Here $k=p_{1}+p_{2}=p_{3}+p_{4}$.

Using the relation (5) one obtains:

$$
\begin{gathered}
\Gamma\left(p_{1}, \ldots, p_{4}\right)=\sum_{\substack{i_{1}, i_{2}=0 \\
j_{1}, j_{2}=0}}^{\infty} \kappa^{i_{1}}\left(p_{1}\right) \kappa^{i_{2}}\left(p_{2}\right) \kappa^{j_{1}}\left(p_{3}\right) \kappa^{j_{1}}\left(p_{4}\right) \\
\times\left\{V_{i_{1}, i_{2}, 0} \frac{1}{\kappa(k)} V_{j_{1}, j_{2}, 0}+\right. \\
\sum_{r=1}^{\infty} \kappa^{r-1}(k)\left[V_{i_{1}, i_{2}, 0} V_{j_{1}, j_{2}, r}+V_{i_{1}, i_{2}, r} V_{j_{1}, j_{2}, 0}\right]+ \\
\left.+\sum_{r_{1}, r_{2}=1}^{\infty} \kappa^{r_{1}+r_{2}-1}(k) V_{i_{1}, i_{2}, r_{1}} V_{j_{1}, j_{2}, r_{2}}\right\} .
\end{gathered}
$$

Here $V_{i k r}$ are the coupling constants of the 3-leg vertex.

It is important to remember that the expressions (8) and (7) are identical because they describe the same graph. However, the interpretation of what may be called a subgraph depends on our definition of the notion of reducibility.

Indeed, according to the conventional understanding of one-particle reducibility the graph $\Gamma$ corresponding to the expression (7) is 1PR: it is constructed from two subgraphs (triple effective vertices) connected with one another by the propagator line.

In contrast, the expression (8) can be considered as the sum of two items:

$$
\Gamma=\Gamma_{1}+\Gamma_{2}
$$

where

$$
\begin{aligned}
\Gamma_{1}=\sum_{\substack{i_{1}, i_{2}=0 \\
j_{1}, j_{2}=0}}^{\infty} \kappa^{i_{1}}\left(p_{1}\right) \kappa^{i_{2}}\left(p_{2}\right) \kappa^{j_{1}}\left(p_{3}\right) \kappa^{j_{1}}\left(p_{4}\right) \times & \\
& \times\left[V_{i_{1}, i_{2}, 0} \frac{1}{\kappa(k)} V_{j_{1}, j_{2}, 0}\right]
\end{aligned}
$$


looks like the customary (traditionally defined) 1PR graph, while $\Gamma_{2}$

$$
\begin{aligned}
\Gamma_{2}=\sum_{\substack{i_{1}, i_{2}=0 \\
j_{1}, j_{2}=0}}^{\infty} \kappa^{i_{1}}\left(p_{1}\right) \kappa^{i_{2}}\left(p_{2}\right) \kappa^{j_{1}}\left(p_{3}\right) \kappa^{j_{1}}\left(p_{4}\right) \times & \\
& \times\left\{\sum _ { r = 1 } ^ { \infty } \kappa ^ { r - 1 } ( k ) \left[V_{i_{1}, i_{2}, 0} V_{j_{1}, j_{2}, r}\right.\right. \\
& \left.+V_{i_{1}, i_{2}, r} V_{j_{1}, j_{2}, 0}\right]+ \\
& \left.+\sum_{r_{1}, r_{2}=1}^{\infty} \kappa^{r_{1}+r_{2}-1}(k) V_{i_{1}, i_{2}, r_{1}} V_{j_{1}, j_{2}, r_{2}}\right\}
\end{aligned}
$$

may be considered as the 1PI 4-leg effective vertex (of the tree level!) with coupling constants constructed as products of 3-leg constants of the Hamiltonian level. The disappearance of the propagator line $1 / k$ due to the presence of the "killing" factor $k$ in adjacent vertex (or both vertices) is called the reduction of the line $k$.

Suppose now that we need to know the graph $\Gamma\left(p_{1}, \ldots, p_{4}\right)$ on the mass shell $p_{i}^{2}=m^{2}$ or, the same, we are only interested in its contribution with accuracy up to the terms of order $\kappa\left(p_{i}\right)$. This means that we can put

$$
\kappa\left(p_{i}\right)=0, \quad(i=1, \ldots, 4) .
$$

(but $\kappa(k)=\left(p_{1}+p_{2}\right)^{2}-m^{2} \neq 0$ !). In this case both triple vertices in the graph (10) turn out minimal with respect to all their lines. The same is true with regard to the 4-leg effective vertex (graph (11)). So, the singular (pole) part (10) of the contribution of the initially considered graph (7) can be considered as the true one-particle reducible subgraph, and the regular part (11) - as the induced minimal secondary 4-leg vertex of the tree level (see [2]).

It is not difficult to generalize the above analysis for more complicated cases. The important observation is that the definition of the graph reducibility is connected with the shape of the analytic recording of the graph contribution.

Let us now consider 1PI 2-leg 1-loop graph $S$ conventionally called (self energy) with the external leg momentum $p$. In the framework of effective theory the relevant triple vertices are described by the following Lagrangian (see [1]):

$$
H_{3}=\frac{1}{3 !} \sum_{s=0} \tilde{D}^{j k ; s}: \phi\left(\partial^{[s]} \phi^{j}\right)\left(\partial_{[s]} \phi^{k}\right):,
$$

Here : ... : denotes the normal product, and the shortened notation

$$
\partial^{[s] \stackrel{\text { def }}{=}} \partial^{\mu_{1}} \ldots \partial^{\mu_{s}} .
$$

is used. In momentum space the triple vertex (13) can be written as follows:

$$
V\left(\kappa_{1}, \kappa_{2}, \kappa_{3}\right)=i(2 \pi)^{4} \delta\left(k_{1}+k_{2}+k_{3}\right)
$$

where $D^{i j k}$ are certain combinations of the coupling constants $\tilde{D}^{j k ; s}$. 
The expression for the one-loop self-energy graph in effective theory reads (both external lines are considered incoming):

$$
\begin{aligned}
S\left(p^{2}\right)=C\left(p^{2}, \Lambda\right) \delta(p+q)+\sum_{\substack{i j k=0 \\
l m n=0}}^{\infty} D^{i j k} D^{l m n} \kappa_{k}^{i} \kappa_{q}^{l} \times & \\
& \times \int d r d t \delta(p+r-t) \delta(q+t-r) \kappa_{t}^{j+n-1} \kappa_{r}^{k+m-1} .
\end{aligned}
$$

Here $C\left(p^{2}, \Lambda\right)$ stands for the counterterm series:

$$
C\left(p^{2}\right)=\left[C^{[\log ]}\left(p^{2}\right) \cdot \log \Lambda+\sum_{n=0}^{\infty} C^{[n]}\left(p^{2}\right) \Lambda^{2 n}\right],
$$

$\Lambda$ is the cutoff parameter, and every $C^{[x]}\left(p^{2}\right)(x=\log , 0,1, \ldots)$ is a power series in $p^{2}$ :

$$
C^{[x]}\left(p^{2}\right)=\sum_{n=0}^{\infty} c_{n}^{[x]} p^{2 n}
$$

It can be shown that the sum in (15) contains the only nontrivial integral - that with $j+n=k+m=$ 0 ; all the others behave as powers of the cutoff parameter $\Lambda$ with coefficients that are just power series in $p^{2}$. This integral reads:

$$
I\left(p^{2}\right)=\delta(p+q)\left[J\left(p^{2}\right)+a_{1} \log \Lambda+a_{2}\right] .
$$

Here

$$
J\left(p^{2}\right) \equiv-\int d r \frac{p^{2}+2 r p}{\left(r^{2}-m^{2}\right)^{2} \cdot\left[(r+p)^{2}-m^{2}\right]}
$$

and $a_{1}, a_{2}$ are just arbitrary constants (they may depend on $m^{2}$ ). All the diverging terms (as well as $a_{1}$ and $\left.a_{2}\right)$ can be absorbed by the yet unfixed counterterm coefficients $c_{n}^{[x]}(x=\log , 1,2, \ldots)$ and the expression (15) can be rewritten as follows

$$
S\left(p^{2}\right)=\sum_{i l=0}^{\infty} D^{i 00} D^{l 00} \kappa_{p}^{i} \kappa_{p}^{l} J\left(p^{2}\right)+\sum_{n=0}^{\infty} c_{n}\left(p^{2}\right)^{n} .
$$

Here $c_{n}$ are the new (finite) counterterm coefficients to be fixed with the help of renormalization prescriptions.

Let us now reorder the terms in (19) as follows:

$$
S\left(p^{2}\right)=\sum_{i=0}^{\infty} G^{i} J\left(p^{2}\right) \kappa_{p}{ }^{i}+\sum_{i=0}^{\infty} \widetilde{d}_{i} \kappa_{p}{ }^{i} .
$$

Here

$$
G^{i}=\sum_{k=0}^{i} D^{k 00} D^{(i-k) 00},
$$

and the coefficients $\widetilde{d}_{i}$ are certain combinations of $c_{n}$ and various degrees of $m^{2}$. These coefficients remain unfixed until one formulates the corresponding RP's. 
The problem is that the number of $\widetilde{d}_{i}$ is infinite. At the same time there are only two physically grounded requirements which can be used as the relevant RP's. First, we need to comply with the requirement

$$
\left.S\left(p^{2}\right)\right|_{p^{2}=m^{2}}=0
$$

This is necessary for fixing the pole position of the two-leg Green function. Second, for the correct normalization of the wave function one needs

$$
\left.\frac{\partial}{\partial p^{2}} S\left(p^{2}\right)\right|_{p^{2}=m^{2}}=0
$$

These two requirements only allow us to fix the constants $\widetilde{d}_{0}$ and $\widetilde{d}_{1}$ :

$$
\begin{gathered}
\widetilde{d}_{0}=-G^{0} J\left(m^{2}\right) . \\
\widetilde{d}_{1}=G^{0} J^{\prime}\left(m^{2}\right)-G^{1} J\left(m^{2}\right) ;
\end{gathered}
$$

the counterterm coefficients $\widetilde{d}_{i}$ with $i=2,3, \ldots$ remain unfixed though they are certainly nonzero.

As shown in [1], the root of the problem lies in erroneous interpretation of what is the two-leg graph in the framework of effective theory. Let us consider this point in more detail.

It is pertinent to note that both the requirements (22) and (23) are based on the result of formal computation of the full propagator $P\left(p^{2}\right)$. Conventionally this is just a summation of the infinite Dyson's chain constructed from the links (2-leg insertions) connected to one another by simple propagators. Every link is considered as the full 1PI 2-leg function $S\left(p^{2}\right)$. At this point it is tacitly implied that every interim propagator is really presented it the chain. This is not always the case in effective theory just because some of them might be "killed" by the corresponding factors stemming from the adjacent vertices. This leads to a mishmash of orders in the perturbation theory based on the loop counting. Also, as shown above, this prevents one to obtain finite S-matrix elements with the help of finite number of RP's.

Let us analyze the calculation of the Dyson's chain for the (conventionally defined as 1PI) full 2-leg function $S$ (conventional "self energy"). One has:

$$
P\left(p^{2}\right)=\pi+\pi S \pi+\pi S \pi S \pi+\ldots=\quad \stackrel{\text { formally }}{=} \frac{\pi}{1-\pi S}=\frac{\pi}{p^{2}-m^{2}-S}
$$

This result is only valid under the condition

$$
\left|\pi\left(p^{2}\right) S\left(p^{2}\right)\right|<1
$$

When this condition is broken one needs to perform the analytic continuation of the right side of (27). In the case in question this condition is certainly broken: this is clearly visible from the expression (20). At the same time, the analytic continuation of the right side looks too difficult (if ever possible) because the pole is not the only singularity. 


\section{The true self energy function}

In the paper [1] it has been suggested an alternative understanding of the meaning of expression (20).

Let us rewrite (20) as follows

$$
S\left(\kappa_{p}\right)=G^{0} \bar{J}\left(\kappa_{p}\right)+d_{0}+\kappa_{p} d_{1}+
$$

$$
+\sum_{j=1}^{\infty} \kappa_{p}^{j} G^{j} \bar{J}\left(\kappa_{p}\right)+\sum_{j=2}^{\infty} \kappa_{p}^{j} d_{j}
$$

Here

$$
\bar{J}\left(\kappa_{p}\right) \equiv J\left(p^{2}\right)-J\left(m^{2}\right),
$$

the integral $J\left(p^{2}\right)$ has been defined in (18), and

$$
d_{0} \equiv 0 ; \quad d_{1} \equiv-G^{0} J^{\prime}\left(m^{2}\right) ;
$$

$$
d_{j} \equiv \widetilde{d}_{j}+J\left(m^{2}\right) . \quad(j \geqslant 2) .
$$

Note that the counterterm coefficients $d_{j}(j=2,3, \ldots)$ remain unfixed.

Let us rewrite now the expression (28) as follows:

$$
S\left(\kappa_{p}\right)=\Sigma\left(\kappa_{p}\right)+\sum_{j=1}^{\infty} \kappa_{p}^{j} R^{j}\left(\kappa_{p}\right)+\sum_{j=2}^{\infty} \kappa_{p}^{j} d_{j} .
$$

Here

$$
R^{j}\left(\kappa_{p}\right) \equiv G^{j} \bar{J}\left(\kappa_{p}\right),
$$

and

$$
\Sigma\left(\kappa_{p}\right) \equiv G^{0}\left(\bar{J}\left(\kappa_{p}\right)-\kappa_{p} \bar{J}^{\prime}\left(\kappa_{p}\right)\right) .
$$

The above-defined function (33) is exactly what has been called in [1] the "true self energy function" (TSE). It meets the conditions of the type (22) and (23). Surely, those conditions only fix the function $\Sigma\left(\kappa_{p}\right)$ itself. They do not allow us to fix completely the form (19) because, first, the second item depends on the non-minimal parameters $G^{i}(i=1,2, \ldots)$ and, second, the last one contains the higher counterterms $d_{i}$ with $i=2,3, \ldots$. Nevertheless, as will be shown below, the form (31) turns out quite sufficient for computing the dressed lines in graphs of the effective scattering theory. The reason is that in this case we are only interested in the renormalization of $S$-matrix elements; the Green functions may contain infinite terms not affecting the amplitudes of physical processes.

So, from this moment we can consider $\Sigma\left(\kappa_{p}\right)$ defined in Eq. (33) as finite correctly normalized function TSF. What about the higher counterterms that are presented in (31)? They are non-minimal. Therefore they can be absorbed by the adjacent vertices of the tree level; the latter ones can be considered as 1-loop n-leg counterterms with $n \geqslant 3$. The problem of fixing such counterterms should be addressed at a later step of the renormalization process.

\section{Analytic reducibility}

The idea of the future analysis is based on the above-discussed notion of line reduction.

It is pertinent to note that the compliance of the TSE function with the conditions of the type (22) and (23) automatically entails the maintenance of those conditions for the full one-loop 2-leg function 
$S\left(\kappa_{p}\right)$. From this it immediately follows that the insertion of $S\left(\kappa_{q}\right)$ in external line of arbitrary $S$-matrix graph can be neglected: such insertions make no influence on the amplitudes of physical processes.

Let us now consider the effect of insertion of $S\left(\kappa_{r}\right)$ in the internal line of the 1PR tree level graph constructed from two effective vertices $V_{1}\left(p_{1}, \ldots, p_{m}, r\right)$ and $V_{2}\left(q_{1}, \ldots, q_{n}, r\right)$ connected to one another by the minimal line $r^{\mathrm{a}}$. The analytic expression for this graph reads:

$$
\begin{aligned}
& \Gamma\left(p_{1}, \ldots, p_{m} ; q_{1}, \ldots, q_{n}\right)= \\
& \quad=V_{1}\left(p_{1}, \ldots, p_{m}, r\right) \frac{1}{\kappa_{r}} S\left(r^{2}\right) \frac{1}{\kappa_{r}} V_{2}\left(q_{1}, \ldots, q_{n}, r\right) .
\end{aligned}
$$

According to the conventional ("graphical") definition this is the 1PR graph. However, as will become clear below, it is more natural to rely upon the alternative ("analytical") definition of what is reducibility (G1PR and A1PR).

Let us substitute in (34) the explicit expression (31). This gives (with account taken of the relation (21)):

$$
\begin{aligned}
& \Gamma(p, q)= \\
& =\frac{V_{1}(p, r)}{\kappa_{r}}\left[\Sigma\left(\kappa_{r}\right)+\sum_{j=1}^{\infty} \kappa_{r}^{j} R^{j}\left(\kappa_{r}\right)+\sum_{j=2}^{\infty} \kappa_{r}^{j} d_{j}\right] \frac{V_{2}(q, r)}{\kappa_{r}}= \\
& \quad=V_{1}(p, r) \frac{1}{\kappa_{r}} \Sigma\left(\kappa_{r}\right) \frac{1}{\kappa_{r}} V_{2}(q, r)+ \\
& \quad+\sum_{j=2}^{\infty} d_{j}\left[V_{1}(p, r) \kappa_{r}^{j-2} V_{2}(q, r)\right]+ \\
& +\sum_{j=2}^{\infty} G^{j} \bar{J}\left(\kappa_{r}\right)\left[V_{1}(p, r) \kappa_{r}^{j-2} V_{2}(q, r)\right]+ \\
& \quad+G_{1}\left[V_{1}(p, r) \frac{\bar{J}}{\kappa_{r}} V_{2}(q, r)\right] .
\end{aligned}
$$

Let us analyze this result term by term.

In the left side of (35) we have the conventionally $1 \mathrm{PR}$ (or, the same G1PR) graph for the process $m \rightarrow n$ with one-loop dressed internal line. In contrast, the analytic form of the same expression may be interpreted as the finite sum of individual graphs constructed from the minimal secondary effective vertices. Some of those graphs are A1PI while another ones - A1PR. Indeed, the first line in the right side of (35)) (the second line in the Eq. (35)) looks like the A1PR graph with the internal line dressed with the 1-loop TSE insertion. The second item in the right side (see the third line in (35)) looks like the sum of local vertices of the counterterm type. Those counterterm vertices have $m+n$ legs and the loop index $I=1$ This index was caused by the reduction of loop inner lines. The third item (the fourth line in (35)) is, in fact, nothing but the initial one-loop graph with both lines $r$ reduced. At last, the last line in (35) is nothing but the initial graph with one of the lines $r$ reduced.

Clearly, the expression (35) is still divergent because the above considered A1PI one-loop L-leg graphs $(L=m+1, n+1, m+n)$ have not been renormalized; the necessary minimal 1-loop counterterms have not been taken into account. This point will be discussed in forthcoming publications. The main ideas have been discussed in [3].

\footnotetext{
${ }^{\text {a }}$ The non-minimal line $r$ presents no interest because it can be reduced independently of the structure of $S\left(\kappa_{r}\right)$.
} 
At the moment it is important to realize that there is no problem with 1-loop dressing of the lines (both external and internal) of effective scattering theory graphs that occur in the case when we rely upon the notion of analytic reducibility. Besides, the meaning of the term "self energy" becomes clear in this case because the Dyson's sum of full A1PI 2-leg graphs gives for 2-leg Green function the expression

$$
G_{2}\left(p^{2}\right)=\frac{1}{\left(p^{2}-m^{2}\right)-\Sigma\left(p^{2}\right)}+Q\left(\kappa_{p}\right)
$$

(here $Q\left(\kappa_{p}\right)$ is a power series in $\kappa_{p}$ such that $Q(0)=0$ ). This expression is quite acceptable from the physical point of view.

We thank M. A. Braun, S. A. Paston and M. I. Vyazovsky for interesting discussions. The work of V.V.V. was partially supported by the Saint Petersburg State University grant N 11.38.189.2014.

\section{References}

[1] Vladimir V. Vereshagin, Phys. Rev. D 89, 125022 (2014).

[2] A. Vereshagin and V. Vereshagin, Phys. Rev. D 69, 025002 (2004).

[3] K. Semenov-Tian-Shansky, A. Vereshagin, and V. Vereshagin, Phys. Rev. D 73, 025020 (2006).

[4] S. Weinberg, The Quantum Theory of Fields, Vol. 1, (Cambridge University Press, Cambridge, 1996).

[5] N. N. Bogoliubov and D. V. Shirkov, Introduction to the Theory of Quantized Fields (WileyInterscience, New York, 1980). 